OPEN ACCESS

Edited by:

Rahul Kashyap,

Mayo Clinic, United States

Reviewed by:

Juan Pablo Domecq,

Mayo Clinic, United States

Vikas Bansal,

Mayo Clinic, United States

${ }^{*}$ Correspondence:

Haiyan Yin

yinhaiyan1867@126.com orcid.org/0000-0002-9680-4219

Jun Lyu

Iyujun2020@jnu.edu.cn orcid.org/0000-0002-2237-8771

tThese authors have contributed equally to this work

Specialty section: This article was submitted to Intensive Care Medicine and Anesthesiology,

a section of the journal

Frontiers in Medicine

Received: 23 February 2021 Accepted: 27 April 2021

Published: 21 May 2021

Citation:

Zhang L, Zhang F, XU F, Wang Z, Ren Y, Han D, Lyu J and Yin H (2021)

Construction and Evaluation of a Sepsis Risk Prediction Model for Urinary Tract Infection. Front. Med. 8:671184. doi: 10.3389/fmed.2021.671184

\section{Construction and Evaluation of a Sepsis Risk Prediction Model for Urinary Tract Infection}

\author{
Luming Zhang ${ }^{1,2+}$, Feng Zhang ${ }^{1+}$, Fengshuo $\mathrm{Xu}^{2,3}$, Zichen Wang ${ }^{4}$, Yinlong Ren ${ }^{1}$, \\ Didi Han ${ }^{2,3}$, Jun $\mathrm{Lyu}^{2 *}$ and Haiyan Yin ${ }^{1 *}$
}

${ }^{1}$ Intensive Care Unit, The First Affiliated Hospital of Jinan University, Guangzhou, China, ${ }^{2}$ Department of Clinical Research, The First Affiliated Hospital of Jinan University, Guangzhou, China, ${ }^{3}$ School of Public Health, Xi'an Jiaotong University Health Science Center, Xi'an, China, ${ }^{4}$ Department of Public Health, University of California, Irvine, Irvine, CA, United States

Background: Urinary tract infection (UTI) is one of the common causes of sepsis. However, nomograms predicting the sepsis risk in UTI patients have not been comprehensively researched. The goal of this study was to establish and validate a nomogram to predict the probability of sepsis in UTI patients.

Methods: Patients diagnosed with UTI were extracted from the Medical Information Mart for Intensive Care III database. These patients were randomly divided into training and validation cohorts. Independent prognostic factors for UTI patients were determined using forward stepwise logistic regression. A nomogram containing these factors was established to predict the sepsis incidence in UTI patients. The validity of our nomogram model was determined using multiple indicators, including the area under the receiver operating characteristic curve (AUC), correction curve, Hosmer-Lemeshow test, integrated discrimination improvement (IDI), net reclassification improvement (NRI), and decision-curve analysis (DCA).

Results: This study included 6,551 UTI patients. Stepwise regression analysis revealed that the independent risk factors for sepsis in UTI patients were congestive heart failure, diabetes, liver disease, fluid electrolyte disorders, APSIII, neutrophils, lymphocytes, red blood cell distribution width, urinary protein, urinary blood, and microorganisms. The nomogram was then constructed and validated. The AUC, NRI, IDI and DCA of the nomogram all showed better performance than traditional APSIII score. The calibration curve and Hosmer-Lemeshow test results indicate that the nomogram was well-calibrated. Improved NRI and IDI values indicate that our nomogram scoring system is superior to other commonly used ICU scoring systems. The DCA curve indicates that the DCA map of the nomogram has good clinical application ability.

Conclusion: This study identified the independent risk factors of sepsis in UTI patients and used them to construct a prediction model. The present findings may provide clinical reference information for preventing sepsis in UTI patients.

Keywords: urinary tract infection, sepsis, MIMIC III database, prognosis, nomogram 


\section{BACKGROUND}

Sepsis and septic shock are common acute critical diseases with very high mortality rates and which affect millions of people worldwide every year. Early detection and appropriate treatment of sepsis can improve the prognosis (1-3). The early clinical manifestations of sepsis are not specific, the disease progresses, and worsens rapidly, and there is currently no effective treatment. These factors lead to the current high mortality rate of sepsis patients $(4,5)$. A comprehensive understanding of the pathogenesis of sepsis would allow targeted prevention and treatment to be carried out more effectively. However, there is currently no theoretical explanations of the pathogenesis of sepsis (4). Therefore, in the process of clinical diagnosis and treatment, controlling the risk factors for infection and prevention of sepsis occurrence should be comprehensively studied.

Infection of the lower respiratory tract is the primary cause of sepsis with the highest mortality, but UTI is the fastestgrowing cause of sepsis (6). Urosepsis is a serious disease caused by organ failure due to a critical urinary tract infection (UTI) (7), possibly caused by a severe community or hospital-acquired UTI (8). Studies have found that $9-31 \%$ of sepsis cases are caused by infections of the urinary and reproductive tracts (9), with urosepsis accounting for $20 \sim 30 \%$ of all sepsis cases (10). Research have showed that diabetes, C-reactive protein, and calculi are risk factors for urinary sepsis development $(11,12)$. Nomograms are graphical tools for determining the probabilities of individual experiencing clinical events based on statistical prediction models (13). However, nomograms that predict the sepsis risk in UTI patients have received little attention. The purpose of our study was to develop a nomogram for predicting sepsis risk in UTI patients and thereby guide clinical practice.

\section{MATERIALS AND METHODS}

\section{Data Source}

All of our data were extracted from the Medical Information Mart for Intensive Care III (MIMIC-III) database, which was established in 2003 under a United States NIH grant by multiple centers. The current (July 2018) version of the MIMIC-III database is version 1.4, which includes data obtained from 2001 to 2012 on more than 58,000 hospitalized patients at the Beth Israel Deaconess Medical Center, including 38,645 adult, and 7,875 neonatal patients. The patient information in this database is anonymous, and so informed consent was not required for this study. The research personnel participated in a series of courses provided by the National Institutes of Health (NIH) and obtained authorization to access the MIMICIII database after completing the required assessment (certificate number 38601114).

\section{Study Population}

We extracted the required data using structured query language in the Navicat Premium version 11.2.7.0. ICD-9 code 5990 was used to extract patients from the MIMIC-III database who were diagnosed with a UTI. Patients younger than 18 years old or who died within $24 \mathrm{~h}$ of entering the ICU were excluded.
The hadm_id identifier of UTI patients was used to extract the following information: age, sex, comorbidities, first laboratory examination results, and APSIII score. Comorbidities (for comorbidities, we evaluated them according to the Elixhauser Comorbidity Index (ECI) in the database, indicate whether the patient was diagnosed with the disease before or after admission.) included congestive heart failure (CHF), hypertension, chronic pulmonary, renal failure, liver disease, metastatic cancer, solid tumors, obesity, urolithiasis, fluid electrolyte disorders, diabetes. The first laboratory test results after admission included levels of white blood cells, neutrophils (NET), lymphocytes (LYM), hemoglobin, hematocrit, platelets, creatinine, urinary white blood cells, urinary red blood cells, urine $\mathrm{pH}$, urinary protein, urinary blood, and urinary ketone, red blood cell distribution width (RDW), and the first pathogenic microorganism culture is positive or negative.

The event outcome was sepsis occurring during hospitalization for UTI. The diagnosis of sepsis is based on the sepsis-3 (4) updated in 2016 by the Society of Critical Care Medicine and the European Society of Intensive Care Medicine, defined as a life-threatening infection combined with an acute increase in Sequential Organ Failure Assessment score $(\mathrm{SOFA} \geq 2)$.

\section{Statistical Analysis}

This study did not include indicators with $>20 \%$ missing values, and the remaining data were filled using multiple imputation. The "mice" package of $\mathrm{R}$ software was used to obtain 10 estimated data sets.

We randomly divided all remaining UTI patients into training (70\%) and validation (30\%) cohorts. The training cohort was used to construct a nomogram and perform internal validation, and the validation cohort was used to perform external validation. Categorical variables were described as frequency and percentage values, and differences between cohorts were determined using the chi-square or Fisher's exact test. The Shapiro-Wilk test was applied to continuous variables to verify whether they conformed to a normal distribution. Continuous variables were described as mean and standard-deviation values or median and interquartile-range values depending on whether or not they conformed to a normal distribution.

Independent risk factors for sepsis in UTI patients were determined using logistic regression. The variables were screened using forward LN stepwise regression. The determined independent prognostic factors were again analyzed using a logistic regression model, and the results were expressed as odds ratios and 95\% confidence intervals (CIs). A nomogram was finally constructed based on the independent prognostic factors to predict sepsis onset in UTI patients.

Multiple indicators were used to internally and externally validate the nomogram. The area under the receiver operating characteristic curve (AUC) was used to evaluate the recognition ability of the nomogram contour map, and this AUC value was compared with that of APSIII. According to the Youden Index, receiver operating characteristic curves were used to determine the optimal cutoff and its sensitivity and specificity. In addition, the integrated discrimination improvement (IDI) and net reclassification improvement (NRI) were used to calculate 
TABLE 1 | Patient characteristics.

\begin{tabular}{|c|c|c|c|}
\hline Variable & Validation cohort & Training cohort & $p$ \\
\hline N & 1,965 & 4,585 & \\
\hline \multicolumn{4}{|l|}{$\operatorname{Sex}(\%)$} \\
\hline Male & 772 (39.3) & $1,752(38.2)$ & 0.428 \\
\hline Female & $1,193(60.7)$ & 2,833 (61.8) & \\
\hline Age & $73.00(60.00,82.00)$ & $73.00(60.00,82.00)$ & 0.378 \\
\hline APSIII & $45.00(34.00,59.00)$ & $45.00(34.00,59.00)$ & 0.573 \\
\hline \multicolumn{4}{|l|}{ Comorbidities } \\
\hline \multicolumn{4}{|l|}{$\begin{array}{l}\text { Congestive heart } \\
\text { failure }(\%)\end{array}$} \\
\hline No & $1,212(61.7)$ & 2,909 (63.4) & 0.184 \\
\hline Yes & 753 (38.3) & $1,676(36.6)$ & \\
\hline \multicolumn{4}{|l|}{ Hypertension (\%) } \\
\hline No & $808(41.1)$ & $1,874(40.9)$ & 0.874 \\
\hline Yes & $1,157(58.9)$ & $2,711(59.1)$ & \\
\hline \multicolumn{4}{|l|}{$\begin{array}{l}\text { Chronic pulmonary } \\
\text { (\%) }\end{array}$} \\
\hline No & $1,484(75.5)$ & 3,466 (75.6) & 0.975 \\
\hline Yes & $481(24.5)$ & $1,119(24.4)$ & \\
\hline \multicolumn{4}{|l|}{ Diabetes (\%) } \\
\hline No & $1,374(69.9)$ & $3,138(68.4)$ & 0.246 \\
\hline Yes & 591 (30.1) & $1,447(31.6)$ & \\
\hline \multicolumn{4}{|l|}{ Renal failure (\%) } \\
\hline No & $1,544(78.6)$ & 3,629 (79.1) & 0.624 \\
\hline Yes & $421(21.4)$ & 956 (20.9) & \\
\hline \multicolumn{4}{|l|}{ Liver disease (\%) } \\
\hline No & $1,738(88.4)$ & $4,112(89.7)$ & 0.150 \\
\hline Yes & 227 (11.6) & 473 (10.3) & \\
\hline \multicolumn{4}{|l|}{ Metastatic cancer (\%) } \\
\hline No & $1,870(95.2)$ & 4,346 (94.8) & 0.565 \\
\hline Yes & $95(4.8)$ & $239(5.2)$ & \\
\hline \multicolumn{4}{|l|}{ Solid tumors (\%) } \\
\hline No & $1,917(97.6)$ & 4,456 (97.2) & 0.444 \\
\hline Yes & $48(2.4)$ & $129(2.8)$ & \\
\hline \multicolumn{4}{|l|}{ Obesity (\%) } \\
\hline No & $1,847(94.0)$ & 4,315 (94.1) & 0.900 \\
\hline Yes & $118(6.0)$ & $270(5.9)$ & \\
\hline \multicolumn{4}{|l|}{ Urolithiasis (\%) } \\
\hline No & 1,957 (99.6) & 4,561 (99.5) & 0.671 \\
\hline Yes & $8(0.4)$ & $24(0.5)$ & \\
\hline \multicolumn{4}{|c|}{ Fluid electrolyte disorders (\%) } \\
\hline No & $1,095(55.7)$ & 2,628 (57.3) & 0.244 \\
\hline Yes & $870(44.3)$ & $1,957(42.7)$ & \\
\hline \multicolumn{4}{|l|}{ First laboratory test } \\
\hline WBC (K/uL) & $10.50(7.70,14.80)$ & $10.50(7.50,14.80)$ & 0.241 \\
\hline Lymphocytes (\%) & $11.00(6.00,17.40)$ & $11.00(6.30,17.60)$ & 0.387 \\
\hline Neutrophils (\%) & $80.90(72.50,87.40)$ & $81.30(72.80,87.80)$ & 0.299 \\
\hline Hematocrit (g/dL) & $33.90(29.70,38.30)$ & $34.10(30.00,38.50)$ & 0.124 \\
\hline Hemoglobin (g/dL) & $11.30(9.80,12.80)$ & $11.40(9.90,12.90)$ & 0.125 \\
\hline RDW (\%) & $14.80(13.80,16.50)$ & $14.90(13.80,16.50)$ & 0.919 \\
\hline Platelet (K/uL) & $\begin{array}{c}235.00(173.00 \\
311.00)\end{array}$ & $\begin{array}{c}240.00(175.00, \\
319.00)\end{array}$ & 0.095 \\
\hline
\end{tabular}

(Continued)
TABLE 1 | Continued

\begin{tabular}{lccc}
\hline Variable & Validation cohort & Training cohort & $\boldsymbol{p}$ \\
\hline Creatinine (mg/dL) & $1.10(0.80,1.80)$ & $1.10(0.80,1.80)$ & 0.656 \\
Urine RBC (\#/hpf) & $5.00(2.00,20.00)$ & $5.00(2.00,20.00)$ & 0.714 \\
Urine WBC (\#/hpf) & $10.00(3.00,50.00)$ & $10.00(2.00,50.00)$ & 0.248 \\
Urine Ph & $5.50(5.00,6.50)$ & $5.50(5.00,6.50)$ & 0.299 \\
Urine blood (\%) & & & \\
$\quad$ Negative & $514(26.2)$ & $1,227(26.8)$ & 0.634 \\
Positive & $1,451(73.8)$ & $3,358(73.2)$ & \\
Urine protein (\%) & & & \\
Negative & $604(30.7)$ & $1,491(32.5)$ & 0.165 \\
Positive & $1,361(69.3)$ & $3,094(67.5)$ & \\
Urine ketone (\%) & & & \\
Negative & $1,259(64.1)$ & $3,020(65.9)$ & 0.170 \\
Positive & $706(35.9)$ & $1,565(34.1)$ & \\
Microorganism (\%) & & & \\
$\quad$ Negative & $1,396(71.0)$ & $3,175(69.2)$ & 0.155 \\
Positive & $569(29.0)$ & $1,410(30.8)$ & \\
Outcome & & & \\
Sepsis (\%) & & & \\
No & & $3,892(84.9)$ & 0.075 \\
Yes & $1,633(83.1)$ & $693(15.1)$ & \\
\hline
\end{tabular}

the performance improvement of the nomogram over the APSIII scoring system. We also constructed a calibration curve and conducted a Hosmer-Lemeshow test to evaluate the calibration of the nomogram. The decision-curve analysis (DCA) curve describes the net benefits and medical interventions from using the nomogram under the guidance of the APSIII, and was used to evaluate the clinical applicability of the nomogram.

$\mathrm{R}$ (version 4.0.3) and SPSS (version 24.0) software were used for the statistical analyses, and $P<0.05$ was considered statistically significant.

\section{RESULTS}

\section{Baseline Characteristics}

The 6,551 patients were divided into 4,585 and 1,965 in the training and validation cohorts, respectively. Females accounted for 61.8 and $60.7 \%$ of the UTI patients in the training and validation cohorts, respectively. The median age in both cohorts was $73.0(60.0,82.0)$ years. Patients with diabetes mellitus accounted for 31.6 and $30.1 \%$ of those in the training and validation cohorts, respectively, while patients with urinary stones accounted for 0.5 and $0.4 \%$ and those with hypertension accounted for 59.1 and $58.9 \%$. The median APSIII score in both cohorts was $45.0(34.0,59.0)$. From the laboratory test results, patients with proteinuria accounted for 67.5 and $69.3 \%$ of those in the training and validation cohorts, respectively, hematuria patients accounted for 73.2 and $73.8 \%$, and sepsis patients accounted for 15.1 and $16.1 \%$. The remaining baseline characteristics are listed in Table 1. 
TABLE 2 | Independent risk factors for sepsis in UTI patients in the multivariate logistic analysis.

\begin{tabular}{|c|c|c|c|c|}
\hline & OR & $95 \% \mathrm{Cl}$ & & $p$ \\
\hline Congestive heart failure & & & & 0.030 \\
\hline No & Reference & & & \\
\hline Yes & 1.22 & 1.02 & 1.47 & \\
\hline Diabetes & & & & $<0.001$ \\
\hline No & Reference & & & \\
\hline Yes & 1.38 & 1.15 & 1.66 & \\
\hline \multicolumn{5}{|l|}{ Liver disease } \\
\hline \multicolumn{5}{|l|}{ No } \\
\hline Yes & 1.53 & 1.19 & 1.97 & $<0.001$ \\
\hline \multicolumn{5}{|l|}{ Fluid electrolyte disorders } \\
\hline \multicolumn{5}{|l|}{ No } \\
\hline Yes & 1.24 & 1.04 & 1.49 & 0.019 \\
\hline \multicolumn{5}{|l|}{ Microorganism } \\
\hline Negative & Reference & & & \\
\hline Positive & 1.25 & 1.04 & 1.51 & 0.018 \\
\hline APSIII & 1.03 & 1.03 & 1.04 & $<0.001$ \\
\hline Neutrophils (\%) & 0.98 & 0.97 & 0.99 & $<0.001$ \\
\hline Lymphocytes (\%) & 0.94 & 0.93 & 0.96 & $<0.001$ \\
\hline RDW (\%) & 1.09 & 1.04 & 1.13 & $<0.001$ \\
\hline \multicolumn{5}{|l|}{ Urine blood } \\
\hline Negative & Reference & & & \\
\hline Positive & 1.37 & 1.08 & 1.76 & 0.010 \\
\hline \multicolumn{5}{|l|}{ Urine protein } \\
\hline Negative & Reference & & & \\
\hline Positive & 1.67 & 1.32 & 2.12 & $<0.001$ \\
\hline
\end{tabular}

\section{Nomogram Construction}

The above-mentioned variables were screened using forward LN stepwise regression. CHF, diabetes, liver disease, fluid electrolyte disorders, APSIII, NET, LYM, RDW, urinary protein, urinary blood, and microorganisms were established as independent risk factors for sepsis in UTI patients during hospitalization. The sepsis risk was 1.382 -fold higher in diabetic than non-diabetic patients (95\% CI $=1.151-1.656), 1.534$-fold higher in patients with than without liver disease ( $95 \% \mathrm{CI}=1.186-1.973), 1.668$ fold higher in patients with than without proteinuria $(95 \% \mathrm{CI}=$ 1.323-2.116), and 1.374-fold higher in patients with than without hematuria ( $95 \% \mathrm{CI}=1.081-1.759)$. The protective factors for sepsis were NET and LYM (see Table 2). These results were used to construct a nomogram for estimating the sepsis risk in UTI patients during hospitalization (Figure 1).

\section{Nomogram Validation}

We compared the ability to predict sepsis in UTI patients between the nomogram and the APSIII scoring system. Figure 2 indicates that the AUC values of the nomogram were 0.775 (95\% CI $=0.757-0.792)$ and $0.756(95 \% \mathrm{CI}=0.730-0.784)$ for the training and validation cohorts, respectively, which were higher than those of the APSIII scoring system. The optimal cutoff for the nomogram in the training cohort was 0.144 , and the sensitivity and specificity were 0.706 and 0.701 , respectively. In the validation cohort, the optimal cutoff was 0.132 , the sensitivity was 0.730 , and the specificity was 0.784 . Compared with the APSIII system, the NRI values of the nomogram were 0.306 (95\% $\mathrm{CI}=0.242-0.419)$ and $0.306(95 \% \mathrm{CI}=0.236-0.406)$ in the training and validation cohorts, respectively; the corresponding IDI values were $0.021(95 \% \mathrm{CI}=0.012-0.030)$ and 0.034 (95\% CI $=0.017-0.051)$. These findings indicate that our nomogram has better recognition ability and is superior to other commonly used scoring systems.

Figure 3 displays the correction curves of the nomogram. The correction curves of the training and validation cohorts were all almost diagonal, and the Hosmer-Lemeshow test results indicated the absence of statistical significance (training cohort: $\chi^{2}=6.950, P=0.642$; validation cohort: $\chi^{2}=11.823, P$ $=0.223$ ), indicating that the nomogram provided a good fit to the data. Finally, we drew a DCA curve to illustrate the clinical applicability of the nomogram and compared it with the APSIII system (Figure 4). Clinical interventions guided by our nomogram had a higher net benefit than other scoring systems when the threshold probability was between 0.1 and 0.8 in both cohorts.

\section{DISCUSSION}

Our study indicated that CHF, diabetes, liver disease, fluid electrolyte disorders, APSIII, NET, LYM, RDW, urinary protein, urinary blood, and microorganisms are independent risk factors for sepsis in UTI patients. These results were used to construct a nomogram for estimating the sepsis risk in UTI patients during hospitalization. The validity of our nomogram model was determined using multiple indicators, including AUC, correction curve, Hosmer-Lemeshow test, IDI, NRI, and DCA. We determined the best cutoff value according to the Yoden index, and also considered the sensitivity and specificity. In practical application, the choice of cutoff value could be weighed according to the risk of misdiagnosis and missed diagnosis.

In some populations, such as critically ill patients, the fatality rate for urine-derived sepsis is $25-60 \%$ (14). The occurrence of sepsis can be reduced through early risk assessment, reasonable antibiotic treatment, and timely targeted treatments (15). Among the complications, CHF, diabetes, liver disease, and electrolyte disturbances were all associated with sepsis. Diabetes was found to be the most common complication relating to UTI (16). The mechanism involves diabetic patients abnormally metabolizing sugar, fat, and protein, and having reduced protein synthesis and accelerated overall metabolism, resulting in reduced synthesis of immunoglobulin, antibodies and complement, reduced LYM conversion rate, and impaired humoral immune function (17). Leukocyte migration, chemotaxis, phagocytosis, and bactericidal ability in the blood were also significantly reduced, and cellmediated immune function was also reduced. Urinary sugar is a good culture medium, provides favorable conditions for bacterial growth and reproduction, and is also conducive to fungal growth (18). The mechanism of agricultural sepsis is therefore greatly enhanced in diabetic patients. 

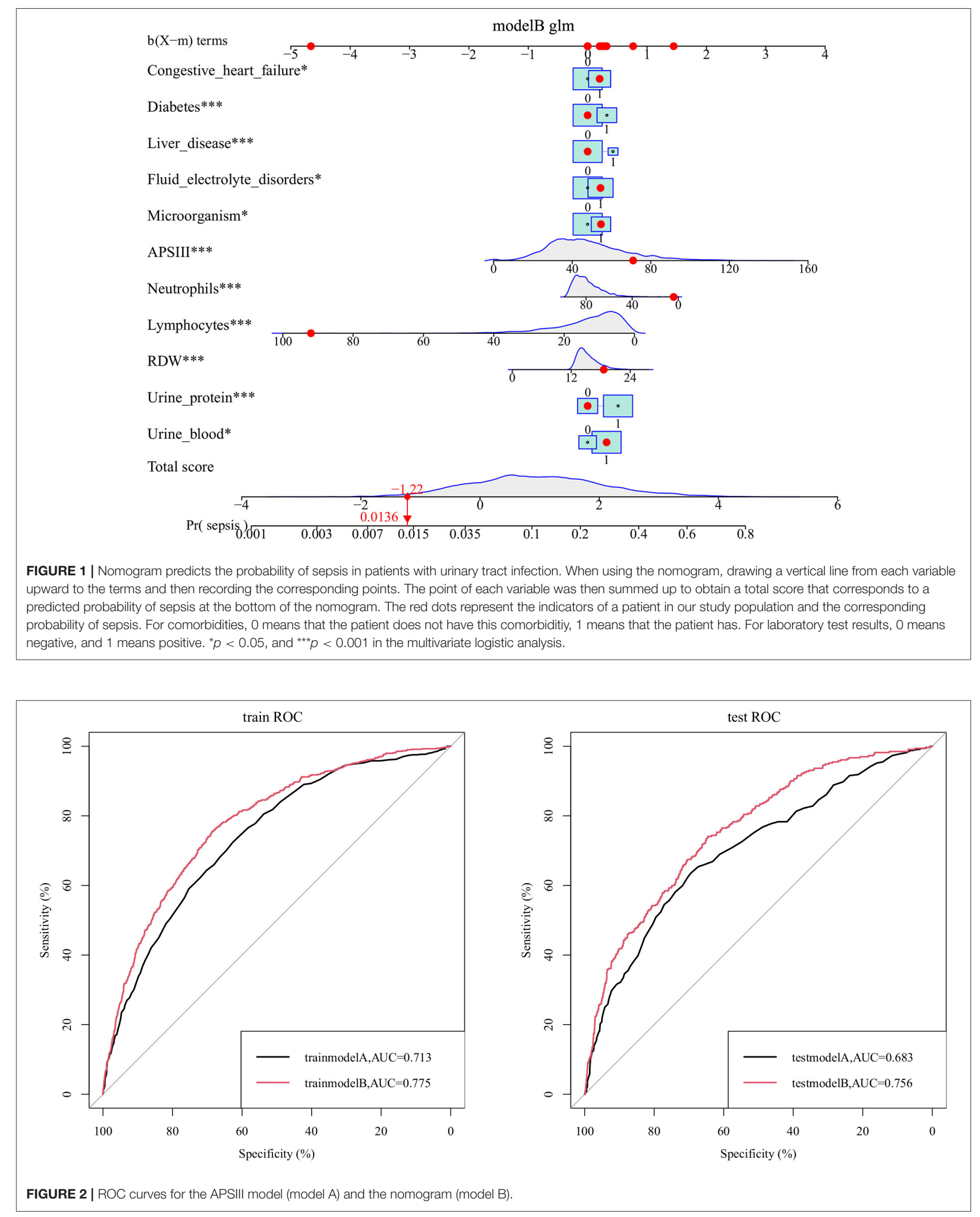

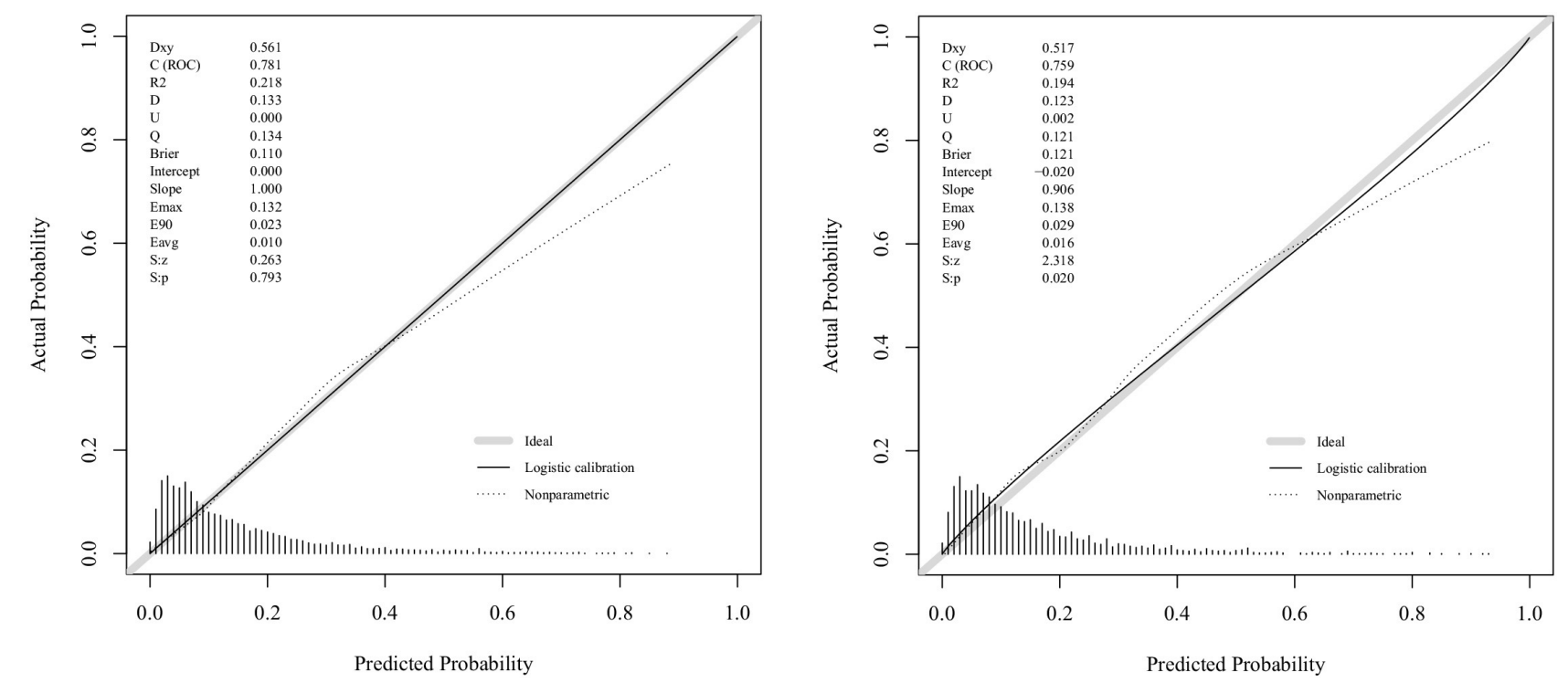

FIGURE 3 | Calibration curves for the validation cohort and the training cohort.
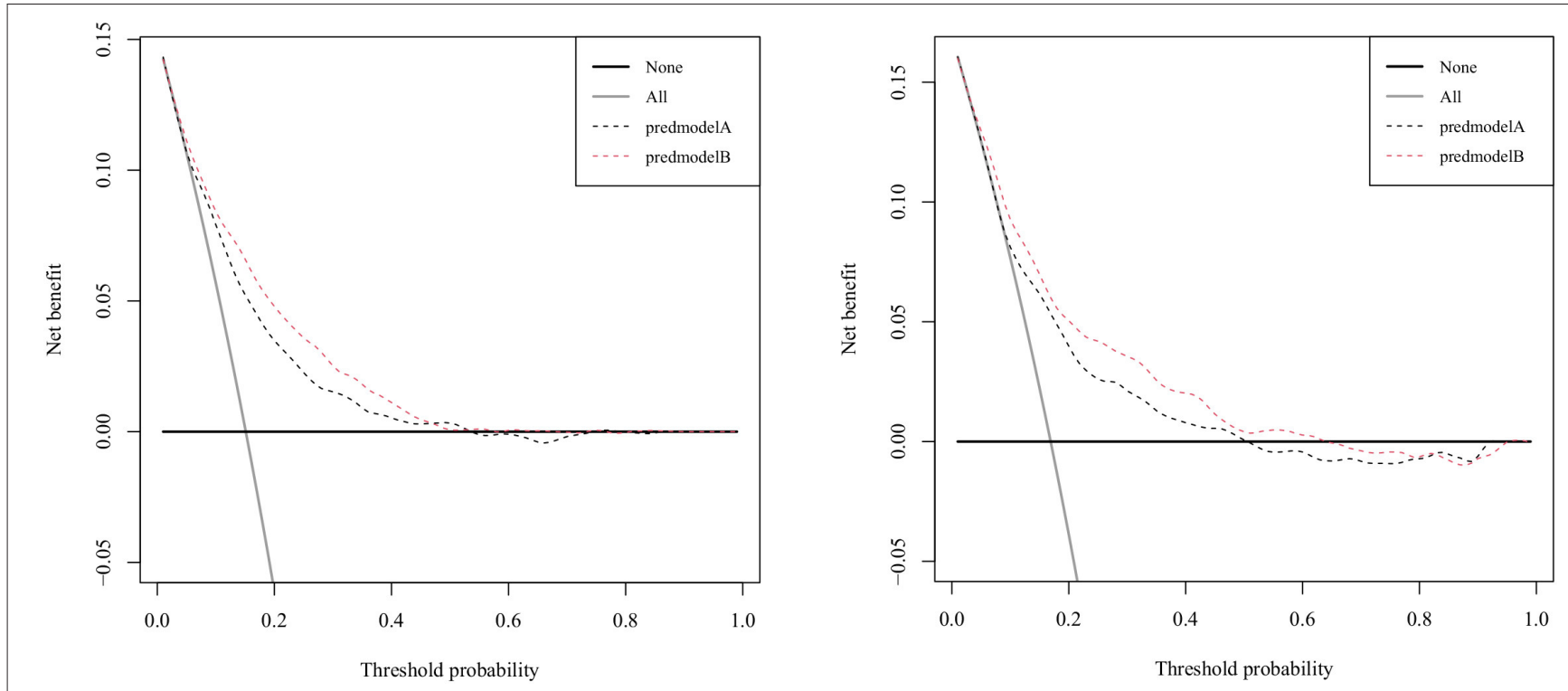

FIGURE 4 | Decision-curve analysis of the validation cohort and the training cohort. Model A represents the APSIII model, and model B represents the nomogram.

Our study showed an increased risk of sepsis in patients with the first positive microbial culture. The pathogen enters the urinary system through retrograde, hematogenous, or lymphatic pathways. Urine-derived sepsis is caused by the pathogen developing further after it enters the blood via the urethra. Patients with pathogen cultures present for the first time should receive clinical attention. Appropriate specimens should be retained for etiological culture before antibiotic use. Antimicrobial agents should include all possible pathogenic bacteria in the initial stage of anti-infective therapy.
NET is one of the important components for the host to fight infection (19), and various mechanisms can be used to enhance the protective immune response (20). LYM is an important component in the immune response of the body, and are an important defense mechanism in biological systems (21). Our results indicated that both NET and LYM were protective factors and that increases in these substances decrease the probability of urine-derived sepsis.

RDW is a clinically-accessible parameter that reflects variations in the size of red blood cells and has mostly being used 
in the diagnosis of circulatory diseases (22). RDW is currently considered to be a strong independent risk factor for human mortality, and many studies have investigated the cardiovascular, cerebrovascular, renal, and other aspects of this parameter (2326). Some studies suggest that RDW has important prognostic value, and can predict hospital and 4-year mortality in critically ill patients (27). RDW is strongly weighted as a risk factor for sepsis in UTI patients in the present nomogram. One study indicated that (28) there is a strong, positive, and independent association between RDW and traditional biomarkers of inflammation, possibly because inflammation reduces the survival rate of red blood cells, leading to differences in red blood cell volumes, and an increase in size heterogeneity among red blood cells. Other studies have indicated that oxidative stress may lead to increases in RDW by increasing erythrocyte turnover, therefore resulting in an association between cell-size inequality and human pathology (29). Patients with increased RDW values should therefore receive specific attention in order to improve their clinical outcomes.

Hematuria is divided into gross and microscopic hematuria, which are common symptoms among UTI patients (30). Proteinuria is positive in $63-83 \%$ of culture-confirmed UTI cases (31). Our results suggest that hematuria and proteinuria are risk factors for sepsis. However, due to the qualitative nature of this study, no conclusions on the quantitative issues of proteinuria and hematuria can be drawn, and the presence of early renal lesions promoting sepsis occurrence remains unclear.

Clinical prediction models were used to investigate the relationship between future outcome events and baseline health status in patients with specific conditions. These can integrate the results of traditional analyses, simplify them through more visually based and convincing presentations, and predict the occurrence probability of certain outcome events through scoring systems. Sepsis and septic shock are major health care problems that affect millions of people around the world every year, and building predictive models for sepsis is clinically important. Some scholars have constructed a nomogram to predict the probability of sepsis after percutaneous nephrolithotomy, and the results showed that patients with complex stones and positive bacteriuria had a significantly higher risk of sepsis after surgery (32). This is a study on urolithiasis, and our study included all patients with UTI in the database, using the history of urolithiasis as one of the study variables. Moreover, we use new indicator such as RDW for the first time to predict the probability of sepsis in patients with urinary tract infection. Other study developed a predictive model that could provide an early risk assessment of sepsis in patients undergoing major hepatobiliary and pancreatic surgery (33). In addition, there are many prognostic models for sepsis patients, for example, one study constructed a predictive model of the 30-day risk of death in patients with sepsis-associated encephalopathy that could be used to assess their prognosis (34). Unlike these studies, to the best of our knowledge, we have constructed the first model that can predict the probability of sepsis in patients with UTI, based on the outcome of the patient's first laboratory examination and comorbidities. This provides a basis for the clinical treatment of patients with UTI. Doctors can use the scoring results of the model to communicate with them and help patients understand the severity of the disease, so that they can jointly make treatment plans and improve their cooperation to prevent the occurrence of sepsis to the greatest extent.

\section{STRENGTHS AND LIMITATIONS OF THE STUDY}

The strength of this study is that we used the MIMIC-III database, a public database containing a large amount of critically ill patient information, which provides strong evidence for our results. Moreover, we developed a nomogram to assess the probability of sepsis by laboratory tests and complications after admission to the ICU in patients with UTI, and demonstrated that the model was effective, something that had not been done before. This study also has some limitations. Although, the number of patients is large, it is a single-center study and lacks external validation.

\section{CONCLUSION}

This study identified the independent risk factors of sepsis in UTI patients and used them to construct a prediction model based. The present findings may provide clinical reference information for preventing sepsis in UTI patients.

\section{DATA AVAILABILITY STATEMENT}

The datasets presented in this study can be found in online repositories. The names of the repository/repositories and accession number(s) can be found below: The data were available on the MIMIC-III website at https://mimic.physionet. org/, http://dx.doi.org/10.13026/C2XW26.

\section{ETHICS STATEMENT}

The study was an analysis of a third-party anonymized publicly available database with pre-existing institutional review board (IRB) approval. Data extracted from the MIMIC III database do not require individual informed consent because MIMIC III database research data is publicly available and all patient data are de-identified.

\section{AUTHOR CONTRIBUTIONS}

LZ created the study protocol, performed the statistical analyses, and wrote the first manuscript draft. FZ conceived the study and critically revised the manuscript. FX assisted with the study design and performed data collection. ZW assisted with study coordination and helped draft the manuscript. YR assisted with data collection and manuscript editing. $\mathrm{DH}$ confirmed the data and assisted with the statistical analyses. JL assisted with manuscript revision and data confirmation. HY contributed to data interpretation and manuscript revision. All authors read and approved the final manuscript. 


\section{FUNDING}

This work was supported by the National Natural Science Foundation of China (Nos. 82072232; 81871585), the Natural Science Foundation of Guangdong

\section{REFERENCES}

1. Angus DC, Linde-Zwirble WT, Lidicker J, Clermont G, Carcillo J, Pinsky MR. Epidemiology of severe sepsis in the United States: analysis of incidence, outcome, and associated costs of care. Crit Care Med. (2001) 29:1303-10. doi: 10.1097/00003246-200107000-00002

2. Martin GS, Mannino DM, Eaton S, Moss M. The epidemiology of sepsis in the United States from 1979 through 2000. N Engl J Med. (2003) 348:1546-54. doi: 10.1056/NEJMoa022139

3. Thompson K, Venkatesh B, Finfer S. Sepsis and septic shock: current approaches to management. Intern Med J. (2019) 49:160-70. doi: 10.1111/imj.14199

4. Singer M, Deutschman CS, Seymour CW, Shankar-Hari M, Annane D, Bauer $\mathrm{M}$, et al. The third international consensus definitions for sepsis and septic shock (Sepsis-3). JAMA. (2016) 315:801-10. doi: 10.1001/jama.2016.0287

5. Cecconi M, Evans L, Levy M, Rhodes A. Sepsis and septic shock. Lancet. (2018) 392:75-87. doi: 10.1016/S0140-6736(18)30696-2

6. Motzkus CA, Luckmann R. Does Infection Site Matter? A Systematic Review of Infection Site Mortality in Sepsis. J Intensive Care Med. (2017) 32:473-79. doi: 10.1177/0885066615627778

7. Pletz MW, Weis S, Forstner C, Wagenlehner F. [Urosepsis]. Med Klin Intensivmed Notfmed. (2018) 113:143-56. doi: 10.1007/s00063-018-0406-1

8. Wagenlehner FM, Pilatz A, Weidner W. Urosepsis-from the view of the urologist. Int $J$ Antimicrob Agents. (2011) 38:51-7. doi: 10.1016/j.ijantimicag.2011.09.007

9. Levy MM, Artigas A, Phillips GS, Rhodes A, Beale R, Osborn T, et al. Outcomes of the surviving sepsis Campaign in intensive care units in the USA and Europe: a prospective cohort study. Lancet Infect Dis. (2012) 12:919-24. doi: 10.1016/S1473-3099(12)70239-6

10. Bonkat G, Cai T, Veeratterapillay R, Bruyère F, Bartoletti R, Pilatz A, et al. Management of urosepsis in 2018. Eur Urol Focus. (2019) 5:5-9. doi: 10.1016/j.euf.2018.11.003

11. Yamamichi F, Shigemura K, Kitagawa K, Fujisawa M. Comparison between non-septic and septic cases in stone-related obstructive acute pyelonephritis and risk factors for septic shock: a multi-center retrospective study. J Infect Chemother. (2018) 24:902-6. doi: 10.1016/j.jiac.2018.08.002

12. Hsiao CY, Chen TH, Lee YC, Hsiao MC, Hung PH, Chen YY, et al. Urolithiasis is a risk factor for uroseptic shock and acute kidney injury in patients with urinary tract infection. Front Med. (2019) 6:288. doi: $10.3389 /$ fmed.2019.00288

13. Zheng Z, Zhang Y, Zhang L, Li Z, Wu X, Liu Y, et al. A nomogram for predicting the likelihood of lymph node metastasis in early gastric patients. BMC Cancer. (2016) 16:92. doi: 10.1186/s12885-016-2132-5

14. Wagenlehner FM, Pilatz A, Naber KG, Weidner W. Therapeutic challenges of urosepsis. Eur J Clin Invest. (2008) 38 (Suppl. 2):45-9. doi: 10.1111/j.1365-2362.2008.02008.x

15. Rhodes A, Evans LE, Alhazzani W, Levy MM, Antonelli M, Ferre R, et al. Surviving sepsis campaign: international guidelines for management of sepsis and septic shock: 2016. Intensive Care Med. (2017) 43:304-77. doi: 10.1007/s00134-017-4683-6

16. Fünfstück R, Nicolle LE, Hanefeld M, Naber KG. Urinary tract infection in patients with diabetes mellitus. Clin Nephrol. (2012) 77:40-8. doi: $10.5414 / \mathrm{CN} 107216$

17. Geerlings SE. Urinary tract infections in patients with diabetes mellitus: epidemiology, pathogenesis and treatment. Int J Antimicrob Agents. (2008) 31:S54-7. doi: 10.1016/j.ijantimicag.2007.07.042

18. Nicolle LE. Urinary tract infections in special populations: diabetes, renal transplant, HIV infection, and spinal cord injury. Infect Dis Clin North Am. (2014) 28:91-104. doi: 10.1016/j.idc.2013.09.006

19. van Rees DJ, Szilagyi K, Kuijpers TW, Matlung HL, van den Berg TK. Immunoreceptors on neutrophils. Semin Immunol. (2016) 28:94-108. doi: 10.1016/j.smim.2016.02.004
Province (No. 2018A030313058), the Technology and Innovation Commission of Guangzhou Science, China (No. 201804010308), and Guangdong Yiyang Healthcare Charity Foundation (No. KT-WSQ2020015).

20. Liew PX, Kubes P. The neutrophil's role during health and disease. Physiol Rev. (2019) 99:1223-48. doi: 10.1152/physrev.00012.2018

21. Alam R, Gorska M. 3. Lymphocytes. J Allergy Clin Immunol. (2003) 111(Suppl. 2):S476-85. doi: 10.1067/mai.2003.121

22. Salvagno GL, Sanchis-Gomar F, Picanza A, Lippi G. Red blood cell distribution width: a simple parameter with multiple clinical applications. Crit Rev Clin Lab Sci. (2015) 52:86-105. doi: 10.3109/10408363.2014. 992064

23. Fava C, Cattazzo F, Hu ZD, Lippi G, Montagnana M. The role of red blood cell distribution width (RDW) in cardiovascular risk assessment: useful or hype? Ann Transl Med. (2019) 7:581. doi: 10.21037/atm.2019. 09.58

24. Wang RR, He M, Ou XF, Xie XQ, Kang Y. The predictive value of RDW in AKI and mortality in patients with traumatic brain injury. J Clin Lab Anal. (2020) 34:e23373. doi: 10.1002/jcla.23373

25. Guo J, Qin Q, Hu H, Zhou D, Sun Y, Deng A. Red cell distribution width (RDW) as a prognostic tool in burn patients. Clin Lab. (2016) 62:1973-8. doi: 10.7754/Clin.Lab.2016. 160222

26. Mohindra R, Mishra U, Mathew R, Negi NS. Red cell distribution width (RDW) index as a predictor of severity of acute ischemic stroke: a correlation study. Adv J Emerg Med. (2020) 4:e24. doi: 10.22114/ajem.v0i0.257

27. Han YQ, Yan L, Zhang L, Ouyang PH, Li P, Goyal H, et al. Red blood cell distribution width provides additional prognostic value beyond severity scores in adult critical illness. Clin Chim Acta. (2019) 498:62-67. doi: 10.1016/j.cca.2019.08.008

28. Lippi G, Targher G, Montagnana M, Salvagno GL, Zoppini G, Guidi GC. Relation between red blood cell distribution width and inflammatory biomarkers in a large cohort of unselected outpatients. Arch Pathol Lab Med. (2009) 133:628-32. doi: 10.5858/133.4.628

29. Kohen R, Nyska A. Oxidation of biological systems: oxidative stress phenomena, antioxidants, redox reactions, and methods for their quantification. Toxicol Pathol. (2002) 30:620-50. doi: 10.1080/01926230290166724

30. Margulis V, Sagalowsky AI. Assessment of hematuria. Med Clin North Am. (2011) 95:153-59. doi: 10.1016/j.mcna.2010.08.028

31. Carter JL, Tomson CR, Stevens PE, Lamb EJ. Does urinary tract infection cause proteinuria or microalbuminuria? A systematic review. Nephrol Dial Transplant. (2006) 21:3031-7. doi: 10.1093/ndt/gfl373

32. Wang J, Mi Y, Wu S, Shao $\mathrm{H}$, Zhu L, Dai F. Impact factors and an efficient nomogram for predicting the occurrence of sepsis after percutaneous nephrolithotomy. Biomed Res Int. (2020) 2020:6081768. doi: $10.1155 / 2020 / 6081768$

33. Zhang H, Meng F, Lu S. Nomograms predicting the occurrence of sepsis in patients following major hepatobiliary and pancreatic surgery. Gastroenterol Res Pract. (2020) 2020:9761878. doi: 10.1155/2020/9761878

34. Yang Y, Liang S, Geng J, Wang Q, Wang P, Cao Y, et al. Development of a nomogram to predict 30-day mortality of patients with sepsis-associated encephalopathy: a retrospective cohort study. J Intensive Care. (2020) 8:45. doi: 10.1186/s40560-020-00459-y

Conflict of Interest: The authors declare that the research was conducted in the absence of any commercial or financial relationships that could be construed as a potential conflict of interest.

Copyright (c) 2021 Zhang, Zhang, Xu, Wang, Ren, Han, Lyu and Yin. This is an open-access article distributed under the terms of the Creative Commons Attribution License (CC BY). The use, distribution or reproduction in other forums is permitted, provided the original author(s) and the copyright owner(s) are credited and that the original publication in this journal is cited, in accordance with accepted academic practice. No use, distribution or reproduction is permitted which does not comply with these terms. 